\title{
A COMMODITY PRICE PROCESS WITH A UNIQUE CONTINUOUS INVARIANT DISTRIBUTION HAVING INFINITE MEAN
}

\author{
BY \\ Eugenio S. A. BobenRIETH H. \\ Departamento de Economía, Universidad de Concepción, Casilla 1987, Concepción, Chile; ebobenri@udec.cl \\ JuAN R. A. BOBENRIETH H. \\ Departamento de Matemática, Universidad del Bío-Bío, Casilla 5-C, Concepcion, Chile; jbobenri@ubiobio.cl \\ AND \\ BRIAN D. WRIGHT \\ Department of Agricultural and Resource Economics, 207 Giannini Hall, University of California, \\ Berkeley, CA94720-3310; wright@are.berkeley.edu
}

Revised March 8, 2001

We present a model of a storable commodity with a price process different from those usually associated with agricultural commodities or exhaustible resources. This model has positive probability of zero output. For consumption equal to zero, price is infinite. In any initial period with finite price, the path of expected prices, conditional on current information, approaches infinity at a rate determined by the cost of storage, and the variance also approaches infinity. Price is expected to fall with probability one in finite time. Successive price realizations are always correlated, storage remains strictly positive, and expected consumption exceeds a strictly positive bound. There is a continuous limiting invariant distribution of price, with infinite mean.

KEYWORDS: Commodities, price, storage, speculation, bubble 


\title{
A COMMODITY PRICE PROCESS WITH A UNIQUE CONTINUOUS INVARIANT DISTRIBUTION HAVING INFINITE MEAN
}

\author{
By Eugenio S. A. Bobenrieth H., JuAn R. A. BobenRieth H. AND BRian D. WRight ${ }^{1}$
}

\section{INTRODUCTION}

IN STANDARD COMPETITIVE MODELS of a storable commodity in the tradition of Gustafson (1958) (for example, Samuelson (1971), Gardner (1979), Newbery and Stiglitz (1981), Wright and Williams (1982, 1984), Scheinkman and Schechtman (1983), Deaton and Laroque (1992)), expected price for the following period exceeds current price by the marginal cost of storage, whenever stocks are positive. The models fit the stylized fact that even after the worst production realization there is always a finite market-clearing price, via the adoption of one of two restrictions: demand is specified so that price at consumption equal to minimum harvest is finite, or the probability of minimum harvest is zero.

Models incorporating either restriction have implications inconsistent with three additional stylized facts of commodity markets. First, they generate stock-outs with positive probability, contrary to the observation of continually positive stocks for many commodities. Second, prices above the stock-out threshold do not exhibit the positive serial correlation observed over the full range of sample prices in many markets (Deaton and Laroque 1992, 1996). Third, price expected for the next period is never below spot price, in contrast to inferences from futures market observations at low stock levels in empirical "supply of storage" studies in the tradition of Working (1934). One way to make the model consistent with all four stylized facts would be to impose a sufficiently large marginal "convenience yield” at small stock levels. Disadvantages of 
this approach include the lack of a clear theoretical basis and the absence of direct evidence of convenience yield. As an alternative, Bobenrieth and Wright (2000) derive typical supply of storage behavior as an aggregation phenomenon, in a variant of the standard model that assumes no convenience yield but includes marketing costs as a function of stocks and sales.

Here we show that the first three of the above four stylized facts of commodity markets can be replicated by the behavior of a variant of the canonical model of Scheinkman and Schechtman (1983), if it is specified such that zero output has positive probability, and price at zero consumption is infinite. In the limiting behavior of this model, with probability one, stocks are strictly positive and price is finite and serially correlated at all finite price levels. But the path of expected prices, conditional on current information, approaches infinity, rising at a rate equal to the cost of storage. There is a unique invariant limiting distribution of price, with infinite mean.

The occurrence of expected price spreads that are always at full storage cost contrasts with the implications of the standard models, and of futures price profiles for many commodities, under assumptions of risk neutrality, rationality, and zero transaction cost. Thus far our characterization of price behavior might make it seem analogous to that of an exhaustible resource (cf. Scheinkman and Schechtman (1983, p. 433)). But price, though always rising in expectation, falls with probability one in finite time, behavior often associated with price “bubbles.” Given a finite initial price, the variance of price approaches infinity. In further contrast with exhaustible-resource behavior, the invariant price distribution is continuous, and expected consumption in any future period exceeds a strictly positive constant, independent of time. Our model violates Samuelson’s (1971) transversality condition that discounted expected price goes to zero in the limit. 
In the next section we outline the model. In Section 3 we address the behavior of available supply and the nature of its invariant distribution. The implications for the invariant distributions of consumption and price are addressed in Section 4, followed by a conclusion.

\section{THE MODEL}

As in previous studies of commodity storage, we model a competitive market for a single storable consumption commodity. Time is discrete. All agents have rational expectations.

In general, we follow the model of Scheinkman and Schechtman (1983). In this paper our focus is on the special case in which production is subject to one common exogenous i.i.d. multiplicative disturbance $\omega \in K \equiv[0, \bar{\mu}], \quad 0<\bar{\mu}<+\infty$, and $\omega$ has a mixed discrete-continuous distribution with a single atom, at zero. More precisely, the distribution of $\omega$ is of the form $\alpha L_{1}+(1-\alpha) L_{2}$, where $\alpha \in(0,1), L_{1}$ is a discrete distribution with a unique atom at zero, and $L_{2}$

is an absolutely continuous distribution with derivative $m$ specified as in Scheinkman and Schechtman (1983, p.436): the support of $m$ is $K$ and $m$ is continuous on $K$.

Assume that there is a continuum of identical producers, and a continuum of identical consumers, both of total measure one. There is a one-period lag between the producers' choice of effort $\lambda \geq 0$ and output $\omega^{\prime} \lambda$, where $\omega^{\prime}$ is next period's productivity shock. Cost of effort is given by a function $g: \mathbb{R}_{+} \rightarrow \mathbb{R}_{+}$, with $g(0)=0, g^{\prime}(0)=0$, and $g^{\prime}(\lambda) \geq 0, g^{\prime \prime}(\lambda)>0$ for all $\lambda>0$. The producer can store output from one period to the next. The amount stored is $x \geq 0$. Storage cost is given by a function $\phi: \mathbb{R}_{+} \rightarrow \mathbb{R}_{+}$, with $\phi(0)=0, \phi^{\prime}(0) \in \mathbb{R}$, and $\phi^{\prime}(x)>0$, $\phi^{\prime \prime}(x)>0$ for all $x>0$. Given storage $x$ and effort $\lambda$, the next period's total available supply is $z^{\prime} \equiv x+\omega^{\prime} \lambda$. Producers are risk neutral and have a constant discount factor $\delta, 0<\delta<1$. 
Utility is a function $U: \mathbb{R}_{+} \rightarrow \mathbb{R}_{+}$. $U$ is continuous in $\mathbb{R}_{+}$, continuously differentiable in ] $0,+\infty\left[, \quad\right.$ strictly concave, and satisfies $U(0)=0, \quad U^{\prime}(c)>0 \quad \forall c>0$, $U^{\prime}(0)=\lim _{c \rightarrow 0+} U^{\prime}(c)=+\infty$, and $\lim _{c \rightarrow \infty} U(c) \leq B<+\infty$. Consumer's demand is $f=U^{\prime}$. Note that $U(c)=\int_{0}^{c} f(u) d u$, and that total revenue $c f(c)$ is bounded.

The perfectly competitive market yields the same solution as the surplus maximization problem. The Bellman equation for the surplus problem is:

$$
\begin{aligned}
& v(z)=\max _{x, \lambda}\left\{U(z-x)-\phi(x)-g(\lambda)+\delta E\left[v\left(z^{\prime}\right)\right]\right\}, \\
& \text { s.t. } \quad z^{\prime}=x+\omega^{\prime} \lambda, \\
& \quad x \geq 0, \quad z-x \geq 0, \quad \lambda \geq 0,
\end{aligned}
$$

where $E[$.$] denotes the expectation with respect to next period's production shock \omega^{\prime}$.

Standard results apply, establishing that $v$ is continuous, strictly increasing, strictly concave, and that the optimal policy functions $x(z)$ and $\lambda(z)$ are single valued and continuous. Consumption and price are given by the functions $c(z) \equiv z-x(z), p(z) \equiv f(z-x(z))$.

The policy functions satisfy the Euler conditions:

$$
\begin{array}{ll}
f(z-x(z))+\phi^{\prime}(x(z)) \geq \delta E\left[v^{\prime}\left(x(z)+\omega^{\prime} \lambda(z)\right)\right], & \text { with eq. if } x(z)>0, \\
g^{\prime}(\lambda(z)) \geq \delta E\left[\omega^{\prime} v^{\prime}\left(x(z)+\omega^{\prime} \lambda(z)\right)\right], & \text { with eq. if } \lambda(z)>0,
\end{array}
$$

and the envelope condition $v^{\prime}(z)=f(z-x(z))$.

Our assumption on the distribution of the $\omega^{\prime} s$, and the first arbitrage condition above, imply that if $z>0$, then $z^{\prime}>0$ and $x\left(z^{\prime}\right)>0$. Thus, if current supply is positive, in any later period expected price exceeds current price. Note that $p(0)=f(0)=+\infty$. Contemplation of such price 
behavior naturally raises issues of stationarity, which we approach by addressing the behavior of available supply, and then the implications for consumption and price.

\section{THE BEHAVIOR OF AVAILABLE SUPPLY}

For available supply $z \geq 0$ and disturbance $\omega^{\prime}=\mu$, the function $h(z, \mu)=x(z)+\lambda(z) \mu$ describes the value of $z^{\prime}$. Note that storage $x(z)$ is continuous and strictly increasing with $z$, $\lambda(z)$ is decreasing with $z, x(0)=0$, and that $g^{\prime}(0)=0$ implies $\lambda(z)>0$ for each $z \geq 0$. The results of Scheinkman and Schechtman (1983, section 5) in Lemmata 1, 2, 3, and 4, hold under our assumptions. In particular, the facts that storage costs are positive and strictly convex imply the existence of an upper bound on storage, $\sup _{z \geq 0} x(z)<+\infty$. Then a suitable state space is $S \equiv[0, \bar{z}]$, where $\bar{z} \equiv \sup _{z \geq 0} x(z)+\lambda(0) \bar{\mu}$. Storage takes values in the set $[0, \bar{x}]$, where $x(0)=0$ and $\bar{x} \equiv x(\bar{z})$, labor supply takes values in the set $[\underline{\lambda}, \bar{\lambda}]$, where $\underline{\lambda} \equiv \lambda(\bar{z})>0$ and $\bar{\lambda} \equiv \lambda(0)$.

Consumption takes values in the set $[0, \bar{c}]$, where $c(0)=0$ and $\bar{c} \equiv c(\bar{z})$. Without loss of generality, let the initial value of $z$ be in $S$.

The transition probability of available supply is

$$
\begin{aligned}
\operatorname{Prob} & {\left[z^{\prime} \leq a^{\prime} \mid z=a\right]=\operatorname{Prob}\left[x(a)+\omega^{\prime} \lambda(a) \leq a^{\prime}\right] } \\
& =\operatorname{Prob}\left[\omega^{\prime} \leq \frac{a^{\prime}-x(a)}{\lambda(a)}\right]=\left\{\begin{array}{ccc}
0, & \text { if } & a^{\prime}<x(a), \\
\alpha+(1-\alpha) \int_{0}^{\frac{\left[a^{\prime}-x(a)\right]}{\lambda(a)}} m(\mu) d \mu, & \text { if } & a^{\prime} \geq x(a) .
\end{array}\right.
\end{aligned}
$$

Let $M$ be the c.d.f. for the density $m$. If the distribution of $z$ is $G$, the distribution of $z^{\prime}$ is 
(2) $\quad T^{*}(G)\left(a^{\prime}\right)=\left\{\begin{array}{lll}\alpha G\left(x^{-1}\left(a^{\prime}\right)\right)+(1-\alpha) \int_{S} M\left(\frac{a^{\prime}-x(a)}{\lambda(a)}\right) d G(a), & \text { if } & 0 \leq a^{\prime}<\bar{x}, \\ \alpha+(1-\alpha) \int_{S} M\left(\frac{a^{\prime}-x(a)}{\lambda(a)}\right) d G(a), & \text { if } \quad a^{\prime} \geq \bar{x} .\end{array}\right.$

Let $\mathcal{G}(S)$ be the set of all c.d.f.'s with support on $S$. Equation (2) defines the adjoint operator $T^{*}: \mathcal{G}(S) \rightarrow \mathcal{G}(S)$. A fixed point of $T^{*}$ is an invariant distribution.

The following Theorem indicates that from any initial available supply, the distribution of available supply approaches a unique distribution, which is a global attractor.

THEOREM: There exists a unique fixed point $G_{*}$ of $T^{*}$. Furthermore, if $G$ is an arbitrary initial distribution on $z$, then $\left(T^{*}\right)^{t}(G)$ converges uniformly to the invariant distribution $G_{*}$ (as $t \rightarrow+\infty)$, at a geometric rate.

ProOF: Note that the transition probability (1) can be written as $\alpha P_{1}+(1-\alpha) P_{2}$, where

$$
\begin{gathered}
P_{1}\left(a,\left[0, a^{\prime}\right]\right)=1, \quad P_{2}\left(a,\left[0, a^{\prime}\right]\right)=\int_{x(a)}^{a^{\prime}} \frac{1}{\lambda(a)} m\left(\frac{z^{\prime}-x(a)}{\lambda(a)}\right) d z^{\prime}, \quad \text { if } x(a) \in\left[0, a^{\prime}\right] \\
P_{1}\left(a,\left[0, a^{\prime}\right]\right)=P_{2}\left(a,\left[0, a^{\prime}\right]\right)=0, \text { otherwise. }
\end{gathered}
$$

Let $T_{1}$ and $T_{2}$ be the Markov operators associated with $P_{1}$ and $P_{2}$. The Markov operator associated with the transition probability (1) is $T=\alpha T_{1}+(1-\alpha) T_{2}$.

Let $B(S)$ be the set of all bounded and measurable real valued functions on $S$, and let $C(S)$ be the subset of $B(S)$ consisting of the continuous functions. For each $u \in B(S)$,

$$
T u(a)=\alpha u(x(a))+(1-\alpha) \int_{x(a)}^{\bar{z}} \frac{u\left(z^{\prime}\right)}{\lambda(a)} m\left(\frac{z^{\prime}-x(a)}{\lambda(a)}\right) d z^{\prime} .
$$


From Theorem 4.6 in Futia (1982, p. 394), $T_{2}$ is weakly compact, implying that $T_{2}^{2}$ is compact, and therefore $T_{2}$ is quasi-compact. Observing that $T_{1}$ is linear and continuous, by Theorem 4.10 in Futia (1982, p. 397), we conclude that $T$ is quasicompact. Since $T(C(S)) \subseteq C(S)$, Theorem 3.3 of Futia (1982, p. 389) implies that $T$ is equicontinuous. Considering that the transition probability (1) satisfies the Uniqueness Criterion 2.11 in Futia with respect to the point 0, using Theorem 2.12 in Futia (1982, p. 385) we conclude that there is a unique probability $\gamma_{*}$ satisfying $T^{*}\left(\gamma_{*}\right)=\gamma_{*}$.

Finally, observing that the transition probability (1) also satisfies, with respect to the point 0 , what is called in Futia a Generalized Uniqueness Criterion, Theorems 3.2, 3.6, and 3.7 in Futia (1982, p. 388 and p. 390) imply that given any initial probability measure $\gamma$ on $z$, the sequence $\left\{\left(T^{*}\right)^{t}(\gamma): t \in \mathbb{N}\right\}$ converges in the total variation norm to $\gamma_{*}$, at a geometric rate. Q.E.D.

If $G$ is the unit point mass at the initial value $z$, the conditional distribution of available supply in the next period, $T^{*} G$, has as its support a finite interval with a single atom, of size $\alpha$, at its lower limit $x(z)$, and the distribution of available supply $t$ periods in the future, $\left(T^{*}\right)^{t}(G)$, has a single atom of size $\alpha^{t}$ at the lower limit, $x^{t}(z)$, of its support. The invariant distribution $G_{*}$ has no atoms, and the lower limit of its support is zero.

\section{THE BEHAVIOR OF CONSUMPTION AND PRICE}

In this model the conditional expectation of price $j \in \mathbb{N}$ periods in the future exceeds current price and approaches infinity as $j$ approaches infinity. Is such behavior consistent with a continuous invariant distribution of price? We address this issue by first considering the behavior 
of consumption. We shall then use the inverse consumption demand relation to derive the limiting behavior of price.

Having established that $z$ has a unique invariant distribution with no atoms, and that the system converges to it at a geometric rate given any initial distribution, it follows immediately that the same is true for consumption, labor supply, storage, and price.

Note that the upper bound on the invariant distribution for $z$, call it $z^{u}$, is less than $\bar{z}$, and the upper bound on consumption under the invariant distribution is $c^{u}=c\left(z^{u}\right)<c(\bar{z})=\bar{c}$. We can construct $z^{u}$ as follows. Let $\hat{z}_{0}=\bar{z}$, and define the sequence $\left\{\hat{z}_{n}\right\}$ by

$$
\hat{z}_{n+1}=\max _{z \in\left[0, \hat{z}_{n}\right]}[x(z)+\lambda(z) \bar{\mu}]
$$

Clearly $\hat{z}_{1}<\hat{z}_{0}=\bar{z}$, and since the operator on the RHS is monotone, it follows that $\left\{\hat{z}_{n}\right\}$ is a decreasing sequence. Hence it converges, and the limit is $z^{u}$. Let $z^{\#}$ be the maximizing value when the interval is $\left[0, z^{u}\right]$. Then an upper bound on consumption under the invariant distribution is

$$
c^{u}=c\left(z^{u}\right)=z^{u}-x\left(z^{u}\right)=\left[x\left(z^{\#}\right)+\lambda\left(z^{\#}\right) \bar{\mu}\right]-x\left(z^{u}\right)<\lambda(0) \bar{\mu},
$$

where the inequality uses the facts that $0 \leq z^{\#} \leq z^{u}$, so $x\left(z^{\#}\right) \leq x\left(z^{u}\right)$ and $\lambda\left(z^{\#}\right) \leq \lambda(0)$, and that the cost of effort is a strictly convex function. Accordingly, 0 and $c^{-1}(\lambda(0) \bar{\mu})$ are bounds on the support of the invariant distribution of available supply.

For the discussion that follows in this section, it is convenient to use the subscript $t$ to denote the value of a function or of a variable in period $t$. Consider consumption in time $t$, $c_{t}=c\left(z_{t}\right)=z_{t}-x\left(z_{t}\right)$ 
The following corollary establishes that this model is not an exhaustible resource model, because the conditional expectation of consumption, and the mean of its invariant distribution, have a positive and finite lower bound.

COROLLARY 1: There exists a constant $q>0$ such that

$$
\begin{aligned}
& E\left[c\left(z_{t+j}\right) \mid z_{t}\right] \geq q \quad \forall t \in \mathbb{N} \cup\{0\}, \quad \forall j \in \mathbb{N}, \\
& +\infty>E\left[c\left(z_{t+j}\right)\right] \geq q, \text { and }+\infty>\int_{S} c(z) \gamma_{*}(d z)=\lim _{j \rightarrow+\infty} E\left[c\left(z_{t+j}\right)\right] \geq q .
\end{aligned}
$$

PROOF: Since $c$ is increasing and $\lambda(z) \geq \underline{\lambda}>0$ for each $z \in S$, we conclude that $E\left[c\left(z_{t+j}\right) \mid z_{t}\right] \geq c(\underline{\lambda} \bar{\mu} / 2) \cdot \operatorname{Prob}\left[\omega_{t+j} \geq \bar{\mu} / 2\right] \equiv q>0$. Using the facts that $\left\{\gamma_{t}\right\}$ converges to $\gamma_{*}$ in the total variation norm and that $c \in B(S)$, we conclude that $E\left[c\left(z_{t+j}\right)\right]=\int_{S} c(z) \gamma_{t+j}(d z) \rightarrow \int_{S} c(z) \gamma_{*}(d z)($ as $j \rightarrow+\infty)$.

For price $p_{t}=f\left(c_{t}\right)$, let $H_{t}(y) \equiv \operatorname{Prob}\left[p_{t} \geq y\right]$. Starting from a mass point in price, $H_{t}$ has a mass point at the upper bound of its support, and converges uniformly to a unique stationary distribution $H_{*}$, which is continuous and is a global attractor.

As noted above, if initial available supply $z_{0}$ is positive, then storage is positive for all later periods. In this case, it follows from the arbitrage equations that $E\left[p\left(z_{t}\right) \mid z_{0}\right] \geq \frac{1}{\delta^{t}} p\left(z_{0}\right) \geq \frac{1}{\delta^{t}} p(\bar{z})>0$. If $z_{0}=0, E\left[p\left(z_{t}\right) \mid z_{0}\right]=+\infty$ for all $t \geq 0$. Therefore,
$E\left[p\left(z_{t}\right)\right]=\int_{S} E\left[p\left(z_{t}\right) \mid z_{0}\right] d G_{0} \geq \int_{S} \frac{1}{\delta^{t}} p(\bar{z}) d G_{0}=\frac{1}{\delta^{t}} p(\bar{z}) \rightarrow+\infty(t \rightarrow+\infty)$ 
Thus the mean of the invariant distribution is infinite. Furthermore, if $p\left(z_{0}\right)<+\infty$, the facts that $E\left[p\left(z_{t}\right)\right] \rightarrow+\infty$, that $H_{t}$ converges to $H_{*}$, and that $H_{*}(+\infty)=0$, imply that the variance of $p_{t}$ approaches infinity (as $t \rightarrow+\infty$ ).

Though expected price increases without bound, the probability becomes arbitrarily large that realized price is below its expectation:

Corollary 2: For arbitrary $\varepsilon>0$ and $D>0$, there exist $N \in \mathbb{N}$ and $D^{\prime} \geq D$ such that $t \geq N$ implies that $E\left[p_{t}\right]>D^{\prime}$ and $\operatorname{Prob}\left[p_{t} \geq D^{\prime}\right]<\varepsilon$.

PROOF: Since $\lim _{n \rightarrow+\infty} H_{*}(n)=H_{*}(+\infty)=0$ and $H_{t}$ converges to $H_{*}$ (as $t \rightarrow+\infty$ ), there exists $D^{\prime} \geq D$ and $N_{1} \in \mathbb{N}$ such that $t \geq N_{1} \Rightarrow H_{t}\left(D^{\prime}\right)<\varepsilon$. Observing that $E\left[p_{t}\right] \rightarrow+\infty$ we conclude the proof.

Q.E.D.

This corollary obviously has strong implications for the sampling properties of this price process.

\section{CONCLUSION}

A modest modification of the standard assumptions of storage models results in a new price process distinct from those typically associated with agricultural or exhaustible resources. In this model, there is a (possibly arbitrarily small) positive probability of minimum output and consumption price is infinite at minimum output. We prove that price, consumption, storage and available supply can each have a unique continuous invariant limiting distribution which is a global attractor, and in which, almost surely, each respective variable is positive and finite, and expected price exceeds current price, which is serially correlated. The recurrence of episodes of behavior often associated with price "bubbles," namely sequences of rapidly increasing prices, followed by abrupt price falls, is a feature of the limiting behavior of our price process. 
Departamento de Economía, Universidad de Concepción, Casilla 1987, Concepción, Chile; ebobenri@udec.cl

Departamento de Matemática, Universidad del Bío-Bío, Casilla 5-C, Concepcion, Chile; jbobenri@ubiobio.cl

and

Department of Agricultural and Resource Economics, 207 Giannini Hall, University of California,Berkeley, CA 94720-3310; wright@are.berkeley.edu 


\section{REFERENCES}

Bobenrieth, E. S. A., AND B. D. Wright (2000): “A Model of Supply of Storage and Backwardation with Apparent 'Convenience Yield,'” Working Paper, Department of Agricultural and Resource Economics, University of California, Berkeley.

Deaton, A., And G. Laroque (1992): “On The Behaviour of Commodity Prices,” Review of Economic Studies, 59, 1-23.

(1996): “Competitive Storage and Commodity Price Dynamics,” Journal of Political Economy, 104, 896-923.

FutiA, C. A. (1982): "Invariant Distributions and The Limiting Behavior of Markovian Economic Models,” Econometrica, 50, 377-408.

GARDNER, B. G. (1979): Optimal Stockpiling of Grain. Lexington, MA: Lexington Books.

Gustafson, R. L. (1958): Carryover Levels for Grains, USDA Technical Bulletin 1178. Washington, DC: United States Department of Agriculture.

Newbery, D. M. G., AND J. E. Stiglitz (1981): The Theory of Commodity Price Stabilization: A Study in the Economics of Risk. Oxford: Clarendon.

Samuelson, P. A. (1971): "Stochastic Speculative Price," in Proceedings of the National Academy of Science, February, 68.

SCHEINKMAN, J. A., AND J. SCHECHTMAN (1983): “A Simple Competitive Model with Production and Storage,” Review of Economic Studies, 50, 427-441.

Working, H. (1934): "Price Relations between May and New-Crop Wheat Futures at Chicago since 1885,” Food Research Institute Wheat Studies, 10, 183-228.

Wright, B. D., AND J. C. WiLliams (1982): “The Economic Role of Commodity Storage,” Economic Journal, 92, 596-614. 
— (1984): "The Welfare Effects of the Introduction of Storage," Quarterly Journal of Economics, 99, 169-192. 
${ }^{1}$ We would like to thank, with the usual caveat, an anonymous referee for helpful comments, and the Co-Editor, Nancy L. Stokey, for her insightful suggestions regarding revision of the paper. 